\title{
Révélations transfictionnelles
}

\author{
Richard Saint-Gelais \\ Université Laval
}

La lectrice et le lecteur qu'aurait intrigués le titre de cette brève étude ne s'attendront sans doute pas à y découvrir des secrets que je m'apprêterais à rendre publics, et ils auront bien raison. J'y ferai moins, en effet, des révélations que j'aborderai les liens entre un ensemble de pratiques - celles qu'on peut rassembler sous la bannière de la transfictionnalité - et l'opération, à bien y songer curieuse s'agissant de fiction, de divulgation. Ce choix n'est pas arbitraire, car le dévoilement est, on le verra, assez étroitement lié à notre conception de la transfictionnalité, à ses promesses, à ses ambivalences. Ce sera l'objet de la première partie de cet article : que devons-nous penser de la promesse de nous en apprendre davantage sur les personnages, les 
circonstances ou le monde fictif mis en place dans une œuvre antérieure ? Dans un second temps, j'élargirai la perspective en me demandant ce que les pratiques transfictionnelles nous disent, non sur une fiction, mais sur la fiction et, plus précisément, sur le rapport à cette notion plus problématique qu'il n'y paraît.

D'abord un rappel: j'entends par transfictionnalité «le phénomène par lequel au moins deux textes, du même auteur ou non, se rapportent conjointement à une même fiction, que ce soit par reprise de personnages, prolongement d'une intrigue préalable ou partage d'univers fictionnel » (Saint-Gelais, 2012, p. 7). Cela place d'entrée de jeu la transfictionnalité sur un terrain déjà occupé par une notion mieux connue, l'intertextualité, dont elle se distingue toutefois en ceci qu'elle ne s'affiche pas comme une relation de texte à texte (par citation ou allusion), mais plutôt de fiction à fiction, comme si les textes sur lesquels repose forcément cette relation n'en étaient en quelque sorte que les supports. Devant, disons, Marcel poursuivi par les chiens dans lequel Michel Tremblay (1992) reprend le personnage de Thérèse qui figurait déjà dans ses Chroniques du Plateau-Mont-Royal, l'impression qui domine n'est pas celle d'observer la «coprésence entre deux ou plusieurs textes » (Genette, 1982, p. 8), mais bien de retrouver un personnage que des romans et pièces de Tremblay avaient déjà mis en scène. L'intertextualité opère par prélèvement et montage discursif; la transfictionnalité, elle, consiste à explorer de nouveau un espace diégétique instauré dans une œuvre antérieure, que ce soit pour le développer ou pour jeter sur lui un éclairage différent. Du coup, elle fait des mondes fictifs des territoires plus vastes que ce que chaque œuvre en montre; des territoires au sein desquels les personnages pourraient 
apparemment circuler sans entrave. Le lecteur d'œuvres transfictionnelles est donc amené, dans les faits, à rompre avec le principe structuraliste de la " clôture du texte», qui affirme que ce qu'on peut dire d'une œuvre est circonscrit par cette œuvre, que les silences d'un texte littéraire font partie de son projet esthétique et devraient donc être acceptés comme tels. $\mathrm{Au}$ contraire, la transfictionnalité investit les zones d'ombre des récits et, du coup, prétend multiplier les révélations - à la différence de l'intertextualité, qui ne produit nul effet de ce genre : nouvel indice de l'hétérogénéité de ces deux notions.

Quelques exemples montreront l'étendue et la variété de ces dévoilements.

a) Un récit transfictionnel peut couvrir des portions inédites de la vie d'un personnage. On aura reconnu la formule perfectionnée par Balzac dans La Comédie humaine et qui lui permet de multiplier les coups de sonde dans la «biographie » des personnages. Ainsi, le lecteur qui parcourrait successivement Le Cabinet des Antiques, Le Père Goriot et La Peau de chagrin découvrirait Rastignac à 25 ans au milieu des jeunes loups de la société parisienne, puis à 22 ans à la veille de son ascension sociale, puis au seuil de la trentaine, désabusé et cynique.

b) Un récit peut aussi montrer la manière dont un personnage perçoit une histoire déjà racontée sous un autre angle. Charles Bovary est devenu au fil des ans un habitué de ce genre de manœuvre. Personnage focal des première et troisième parties de Madame Bovary, il est, dans la vaste partie centrale de ce roman, relégué au rang de personnage observé - quand il l'est - à travers la perspective peu amène de son épouse Emma. Cette marginalisation tant focale qu'actantielle, 
combinée à la naïveté du personnage qui ne semble guère s'apercevoir des infidélités de son épouse, explique sans doute la multiplication des dérivés transfictionnels qui restituent son point de vue et, dans certains cas, sa voix, Charles devenant alors narrateur: Charles Bovary, médecin de campagne (Améry, 1978), Monsieur Bovary (Grimaldi, 1991) et Monsieur Bovary ce titre était décidément trop alléchant pour ne pas avoir été trouvé à répétition - d'Antoine Billot (2006). Charles est-il le benêt qui ressort de la version d'Emma? Comment voit-il son épouse? Se pourrait-il qu'il en sache davantage sur sa vie secrète que ce qu'elle imagine ? Sans entrer dans le détail de ces récits, on notera que leur formule même les amène à multiplier les révélations (parfois spectaculaires) et à s'offrir tendanciellement comme des entreprises de réhabilitation d'un personnage mésestimé. Cela soulève une question sur laquelle il nous faudra revenir : celle de la légitimité de telles manœuvres.

c) Il en va de même pour le troisième cas de figure, celui des récits transfictionnels qui se donnent comme la solution d'une énigme posée par un récit antérieur. Jean Echenoz, avec ses deux romans Un an et Je m'en vais (1997, 1999), a mis en place un dispositif assez retors qui repose là-dessus. L'héroïne d'Un an, Victoire, découvre à son réveil le cadavre de son amant Félix étendu dans son lit. Frappée d'une amnésie qui l'empêche de savoir ce qui a pu se passer, elle s'enfuit puis mène une vie d'errance pendant un an - une année à l'issue de laquelle elle croise par hasard Félix, qui n'est donc pas mort et qui l'informe du décès de Louis-Philippe peu de temps après sa disparition, alors qu'elle a pourtant rencontré cette connaissance à quelques reprises au cours de l'année écoulée. Le roman se termine sur ces énigmes: qu'est-il arrivé au juste à Félix ? comment Victoire a-t-elle pu voir vivant un homme qu'on lui dit 
mort? Il faudra lire Je m'en vais, paru deux ans plus tard, pour découvrir les réponses à ces questions (mais aussi quelques nouvelles questions). Or cette découverte a de bonnes chances d'être fortuite: le paratexte de Je m'en vais, soigneusement composé à cet égard, ne suggère aucun lien avec le précédent roman d'Echenoz. Il en découle que nombre de lecteurs d'Un an ignoreront durablement que le mélange de perplexité et de frustration dispensé dans ses dernières pages s'évapore dans un autre texte. La situation de ceux qui prennent connaissance des deux romans n'est pas moins curieuse : certes en mesure de saisir ce qui est arrivé à Félix et à Louis-Philippe, ils réalisent du même coup qu'ils ne doivent cet éclairage qu'à leur choix, pour plusieurs contingent, d'aborder Je m'en vais après Un an.

d) Dernier cas de figure de cette liste (non exhaustive) : la révélation peut, non s'ajouter au récit antérieur en tant que supplément de données diégétiques, point de vue suscitant une manière de stéréophonie narrative ou réponse extérieure à des énigmes narratives, mais se substituer à une portion plus ou moins considérable de ce récit, bouleversant donc ce qu'on savait jusque-là de l'histoire. Il s'établit alors une relation belligérante entre les deux versions, qui se résout en principe au profit de celle qui, tenant compte de l'autre, entend la supplanter: la plus récente, bien sûr, selon ce qu'on pourrait appeler l'« effet de dernier mot». Certains écrivains résolus et habiles parviennent à désamorcer ce mécanisme en élaborant des ensembles transfictionnels labyrinthiques, en multipliant les révélations délibérément problématiques et les contradictions irrésolubles; bref, en refusant au lecteur le confort d'une solution qui parvienne à s'imposer. Une bonne partie de l'œuvre de Robert Pinget, de L'Inquisitoire (1962) à L'Ennemi (1987), obéit à ce schéma affolant où les 
« révélations » se révèlent au bout du compte aussi fragiles que les versions auxquelles elles s'opposent. C'est plus classiquement qu'Arthur Conan Doyle, dans " La maison vide », parvient à «ressusciter» son héros sériel Sherlock Holmes, donné comme mort à l'issue du «Dernier problème » paru dix ans plus tôt (voir Conan Doyle, 2006a et 2006b) : il lui suffit pour cela de montrer que Watson, ami de Holmes et narrateur de ses exploits, s'est laissé berner par la mise en scène qui laissait croire à l'issue fatale du combat entre le détective et le professeur Moriarty - l'objectif de cette manœuvre scripturale étant bien entendu de satisfaire les nombreux lecteurs consternés par l'interruption de la carrière fictive de Holmes, qui dès lors a pu se poursuivre comme si son décès n'avait jamais eu lieu. Telle est la précarité des faits narratifs (Thérien, 1984, p. 169) qu'il suffit d'un trait de plume pour annuler ce qu'un précédent récit établissait pourtant avec fermeté, pour peu du moins que le lecteur accepte ces révélations; on sait par exemple que celles de «La maison vide» ont été fortement débattues dans les cercles holmésologiques (voir Baring-Gould, 1967, p. 320-349).

Au-delà de leurs différences, sur lesquelles je reviendrai, ces manœuvres présentent un point commun: chacune suppose qu'une fiction puisse être prolongée et parfois réécrite de manière plus ou moins spectaculaire, permettant ainsi au lecteur curieux de "découvrir» des faits inédits, voire des dessous insoupçonnés de l'histoire. Charles Bovary aurait tué sa première épouse Héloïse (Laura Grimaldi, Monsieur Bovary) ; Robinson Crusoë, loin de reconstituer une civilisation en miniature sur son île, y aurait croupi jusqu'à l'arrivée d'une 
certaine Suzanne Barton dont le récit diffère passablement du roman de DeFoe (Coetzee, 1988) ; c'est Caroline Sheppard et non son frère James qui aurait assassiné Roger Ackroyd (Christie, 1948 ; Bayard, 1998)... Ce qui est chaque fois battu en brèche, c'est un postulat central (encore que controversé) de la théorie de la fiction qui veut que cette dernière soit incomplète, c'est-à-dire rigoureusement indéterminée dès qu'on s'aventure au-delà de la zone couverte par le récit; l'exemple classique est celui du nombre d'enfants de Lady Macbeth, que la pièce de Shakespeare ne précise pas, mais que le premier continuateur venu pourrait indiquer. Un récit transfictionnel peut donc s'insérer dans les interstices, les «blancs», d'une œuvre antérieure. Sans pour autant les combler: d'une part, parce qu'ils sont innombrables; d'autre part, parce que ce nouveau récit comporte ses propres indéterminations. Le Mademoiselle Bovary de Raymond Jean nous en apprend beaucoup plus sur la vie de Berthe à la filature que ne le fait le roman de Flaubert, où cet événement et ses conséquences ne font l'objet que d'une mention laconique et terrible, terrible parce que laconique: " [La grand-mère de Berthe] est pauvre et l'envoie, pour gagner sa vie, dans une filature de coton » (Flaubert, 1986, p. 425). On peut déplorer que l'impact de cette phrase qui tombe comme une sentence soit dilué par l'expansion que lui donne le récit de Jean, mais on ne saurait opposer la supposée complétude de ce dernier à l'incomplétude de Madame Bovary : cette continuation suscite, comme tout récit, ses propres effets d'indétermination. Jean ne s'en prive d'ailleurs pas, qui met en scène une relation passablement ambiguë entre la jeune Berthe et l'homme plus âgé chez qui elle trouvera refuge : Gustave Flaubert...

Mon accent sur les opérations transfictionnelles m'a amené jusqu'ici à mettre entre parenthèses un paramètre 
pourtant central, surtout lorsqu'il est question de révélation : le critère à la fois pragmatique et institutionnel de l'auteur. Les auteurs de prolongements ou de corrections s'octroient une latitude apparemment limite; cela ne les empêche pas de devoir composer avec une réception qui, dans notre culture, ne considérera pas du même œil les suites selon qu'elles sont, ou non, produites par l'auteur original. Ce dernier a-t-il le dernier mot sur l'univers fictif qu'il a élaboré ? D'autres écrivains - ou même, dans certains cas, des lecteurs - peuvent-ils intervenir sur «sa» fiction? Le terme «auteur», on le sait, renvoie étymologiquement à l'idée d'autorité. Pour la critique littéraire qui la reconnaît - on sait que la question ne fait pas l'unanimité - , cette autorité s'exerce sur le plan sémantique : l'auteur est celui qu'une tenace tradition interprétative voit comme le garant du sens de l'œuvre. La transfictionnalité attire l'attention sur un autre aspect de cette autorité, à savoir la capacité, en principe exclusive, de l'auteur original à établir ce qu'il en est « vraiment » des personnages, de leurs existences et du cadre dans lequel celles-ci se déroulent. Privés de cette autorité, les textes produits par d'autres écrivains seront tendanciellement tenus pour des variations ludiques ou, du moins, sans impact sur la diégèse. Mais on sait que l'empire de l'auteur est une donnée historique, qui a émergé progressivement (le Moyen Âge ne connaissait pas l'auteur au sens moderne du terme) et qui tend à s'estomper dans la culture médiatique au profit d'autres régimes d'autorité fictionnelle.

Il découle de tout cela que l'examen de la transfictionnalité, mais aussi sa lecture, entremêlent des paramètres hétérogènes, relevant de la logique de la fiction, du statut de l'auteur dans une culture donnée, sans négliger des considérations textuelles: des continuateurs talentueux, 
comme Michael Dibdin dans The Last Sherlock Holmes Story (1978) ou Jean Rhys dans Wide Sargasso Sea (1982), peuvent troubler leurs lecteurs au point de les amener à remettre en question la version canonique d'une fiction antérieure. Chacun de ces romans parvient en effet à faire vaciller notre conception de personnages célèbres de la littérature anglaise: Sherlock Holmes serait un psychopathe dangereux; l'entourage antillais de Bertha, la première épouse du Rochester de Jane Eyre, aurait conspiré pour la plonger dans la démence. Ce qui rend ces révélations mémorables, ce n'est pas seulement leur caractère spectaculaire; si c'était le cas, il suffirait de multiplier les affirmations inattendues à propos des héros de fiction: la Félicité d'«Un cœur simple» se livrait à l'espionnage, Roquentin a connu Meursault au lycée... (Je précise que ces exemples sont imaginaires.) Encore faut-il que ces affirmations «collent», ce qui n'a de chance de se produire qu'à la faveur d'un équilibre subtil entre altération et identité : un peu comme l'interprétation, dont elles se rapprochent à certains égards, les révélations transfictionnelles ne peuvent «faire surgir un double fond de la parole» (Foucault, 1988, p.xii) qu'en affirmant autre chose que ce que le récit original donnait à lire, tout en imposant l'idée que cet "autre chose » se trouvait en quelque sorte déjà contenu dans ce récit. Encore faut-il que les lecteurs acceptent ces interventions - en acceptent le détail et le principe. Les textes transfictionnels n'affectent pas les récits originaux, qu'ils laissent intacts, mais les espaces diégétiques qu'ils déploient : des espaces labiles, à la merci de la moindre intervention, et pour cette raison même défendus par un ensemble de conventions qui font des opérations transfictionnelles des pratiques sous surveillance. 
Cela m'amène à considérer, selon un élargissement de la perspective, non plus les révélations ponctuelles que des récits transfictionnels greffent à des fictions antérieures, mais ce que la transfictionnalité nous dit sur notre conception générale de la littérature et de la fiction: sa fonction de révélateur esthétique, en somme, et non plus seulement diégétique. Cet effet, la transfictionnalité le doit à son caractère toujours plus ou moins provocateur, susceptible d'amener à s'interroger sur les présupposés de la lecture. Qu'en est-il de l'autorité de l'auteur? Une fiction est-elle bordée par le texte qui l'instaure? Est-ce succomber à l'illusion référentielle que d'accepter de s'interroger sur la part de la fiction à propos de laquelle le texte demeure silencieux: segments non abordés d'une intrigue, motivations psychologiques de personnages non focalisés, etc. ?

Examinons ces questions à travers les trois opérations transfictionnelles cardinales que sont l'expansion, la version et le croisement. L'expansion prolonge un récit, que ce soit en poursuivant l'histoire au-delà de son terme (ce que désigne l'anglais sequel) ou en relatant ce qui précède son amorce (prequel), ce qui arrive simultanément (expansion parallèle) ou lors des ellipses de ce récit (interpolation). La variété de ces formules et surtout leur importance quantitative font de l'expansion l'opération transfictionnelle majeure, celle à laquelle on pourrait être tenté (à tort) de réduire l'ensemble du domaine. Cette prévalence de l'expansion confirme ce que nous pressentons par ailleurs, à savoir que la fiction relève massivement du récit, du narratif ${ }^{1}$. Or, cela expose la

\footnotetext{
1 Il existe certes des fictions non narratives, par exemple essayistiques ; qu'on songe aux critiques imaginaires de Borges comme «Examen de l'œuvre d'Herbert Quain» (Borges, 1957). Mais, loin de mener à une reconceptualisation de la fiction, de tels textes seront le plus souvent réduits à
} 
transfictionnalité à ce qu'on pourrait appeler le paradoxe du narratif. D'une part, tout récit met en place une dynamique qui emporte le lecteur vers son aval- qu'arrivera-t-il ensuite? et parfois vers son amont: quels événements passés ont pu conduire aux situations qu'on nous présente? Le suspense et l'énigme sont, respectivement, les manifestations les plus visibles de ces vecteurs, de cette «énergie du récit» dont d'innombrables suites et prequels suggèrent qu'elle serait difficile à contenir, tant semble vif le désir d'en apprendre davantage sur les personnages et les circonstances. C'est évidemment ce désir que ces textes entendent satisfaire, avec un succès certes variable: n'oublions pas que la pulsion narrative réside en grande partie dans l'imagination du lecteur, et que la satisfaire revient bien souvent à la décevoir.

Cette déception tient à ce que notre relation au récit demande, d'autre part, que celui-ci soit borné ; que, structure et pas seulement dynamique, il s'agence en fonction des limites qui lui sont imposées : un point de départ qui lui soit origine ; un point d'arrivée donné comme un aboutissement, fût-il délibérément déceptif - et, entre les deux, une série de tensions et de détentes qui en scandent le mouvement. On sait ce que l'effet de configuration du récit doit à ses choix, en particulier à son dénouement et à la signification que celui-ci imprime à l'ensemble du parcours narratif. Les dernières pages de Madame Bovary, marquées par la déchéance des Bovary (suicide d'Emma, dépression et mort de Charles) et par l'ascension sociale parallèle du pharmacien Homais (« Il vient de recevoir la croix d'honneur »), scellent l'ironie pessimiste de

des exceptions fascinantes par rapport à ce qui serait la norme - narrative de la fiction. 
ce roman, moins par leur mention que par leur caractère définitif. Le récit instaure une dynamique tout en travaillant à la juguler; il accrédite l'idée que les personnages ont une existence dont il ne livre qu'un fragment, tout en coupant court aux spéculations auxquelles ces existences peuvent donner lieu. Tel est le prix qu'il demande au lecteur de payer pour apprécier l'art de sa construction.

On voit immédiatement que l'expansion joue la dynamique contre la structure, la pulsion contre la configuration: elle fait sauter le verrou par lequel le récit entendait circonscrire son propre mouvement et lui imposer un sens, classiquement téléologique. Ce qui ne veut pas dire que les textes transfictionnels soient pure dynamique, déploiement narratif que rien ne viendrait arrêter : eux aussi, bien entendu, s'orientent vers un dénouement (ce qui pose d'ailleurs quelques problèmes délicats dans le cas des prequels, dont le terme est fixé d'avance); seules les constellations transfictionnelles particulièrement fournies - on ne compte plus, par exemple, les récits apocryphes consacrés à Sherlock Holmes approchent, asymptotiquement, l'illusion d'une fiction infinie.

Alors que les expansions ajoutent des segments diégétiques au récit original, les versions, elles, opèrent par superposition : elles repassent sur les traces d'un récit antérieur en en changeant la perspective ou en valorisant un personnage secondaire, manœuvres évidemment convergentes puisque la focalisation sur un personnage est, comme on s'en doute bien, l'occasion de faire valoir son point de vue (dans le sens rhétorique du terme) et de le placer au cœur du récit, sinon de l'histoire. On ne s'étonnera donc pas si figurent ici en bonne place les mal-aimés (à réhabiliter) et les «êtres de fuite » (à 
élucider), tels Charles Bovary ou Albertine (voir Rose, 2002). Mais il peut aussi s'agir de personnages créés pour l'occasion, donc absents du récit original ${ }^{2}$, sur lequel ils permettent de jeter un regard extérieur. L'un des emplois les plus brillants de cette dernière formule se trouve dans le Mary Reilly de Valerie Martin (1991), dont le récit est narré par une jeune domestique du docteur Jekyll qui n'est jamais mentionnée dans le roman de Stevenson. Loin de substituer des réponses aux questions, comme dans maints textes transfictionnels, la focalisation sur ce personnage qui ignore tout des activités secrètes de son employeur aboutit à ce résultat étonnant: un roman qui fait surgir des énigmes d'un récit qui s'ingénie à les résoudre. Mary Reilly illustre aussi une autre fonction, fréquente depuis quelque temps, des versions transfictionnelles : contester « de l'intérieur » une fiction canonique en donnant la parole à des représentants de groupes marginalisés ou opprimés, comme l'épouse séquestrée de Rochester (Wide Sargasso Sea), le frère de «l'Arabe» tué par Meursault (Daoud, 2014), les domestiques de Mr Darcy (Baker, 2013), etc.

La « leçon » tacite de ces versions se distingue clairement de celle des expansions, puisqu'elles insistent moins sur l'appétit de savoir qui caractérise notre rapport aux intrigues que sur la dimension rhétorique des récits. Proposer une autre version, ce n'est pas seulement éclairer les lecteurs sur les dessous d'une intrigue qu'ils connaissent déjà : c'est rappeler que les textes narratifs ne se structurent pas seulement sur le plan événementiel, qu'ils sont informés par des points de vue

\footnotetext{
${ }^{2}$ Ce que Terence Parsons (1980, p. 51-52) appelle des « autochtones», par opposition aux « immigrants », personnages (ou autres éléments diégétiques : lieux, événements, etc.) qu'un écrivain de fiction emprunte à la réalité ou à d'autres fictions.
} 
(le récit «neutre » n'existe pas), et donc que modifier ce point de vue revient souvent à remettre en question leurs présupposés et à faire voir le caractère arbitraire de leur options (voir Dubois, 2011). On ne s'étonnera donc pas que, de toutes les formules transfictionnelles, ce soient les versions qui aient la faveur de la critique attachée à la dimension « essayistique » de la fiction : la critique idéologique bien sûr (féministe, postcolonialiste, etc.), mais aussi, plus largement, la critique sensible aux effets des choix narratifs.

Le dernier type d'opérations transfictionnelles que j'aborderai ici est un peu plus surprenant : il consiste à croiser (au moins) deux fictions préexistantes, dont les protagonistes peuvent dès lors interagir au sein d'une intrigue inédite se rapprochant peu ou prou de celles qu'on leur connaissait. Les personnages de Fantômette contre le hibou, un roman pour la jeunesse de Georges Chaulet (1962), se pressent aux portes du cinéma où on s'apprête à projeter Ivanhoé contre Robin des Bois, une «superproduction en chromocolor et panorascope » (Chaulet, 1962, p. 25) : un tel film, s'il était tourné, effectuerait un croisement transfictionnel mettant aux prises deux héros vivant dans des univers apparentés mais distincts. Le plaisir que les spectateurs fictifs de Chaulet escomptent (et que ses lecteurs réels leur envieraient, si la projection n'était finalement annulée à la suite du vol des bobines) tient à cette motivation diégétique (deux héros "médiévaux ») d'un contact qui a en même temps quelque chose de légèrement transgressif. La même combinaison de facteurs caractérise les croisements transfictionnels réels (en tant qu'œuvres bien sûr) que proposent Sherlock Holmes contre Dracula (Estleman, 2000), le Mademoiselle Bovary de Maxime Benoît-Jeannin (1991), où la fille des Bovary rencontre notamment Bouvard et Pécuchet, ou 
encore la bande dessinée The League of Extraordinairy Gentlemen d'Alan Moore et Kevin O'Neill (2002), véritable forum transfictionnel rassemblant de nombreux protagonistes de fictions victoriennes: Mina Harker (Dracula), Dr Jekyll, l'homme invisible de Wells, etc. On voit que les auteurs de ces croisements se donnent volontiers une caution diégétique (en élisant par exemple des personnages d'une même époque) ou auctoriale, la seconde étant souvent une forme indirecte de la première, surtout en paralittérature, où l'auteur est considéré comme l'index d'un univers supposé homogène malgré la segmentation des œuvres. La situation est fort différente en littérature générale où, sauf exception (Balzac, Zola, Tremblay, etc.), il n'est pas attendu d'un écrivain qu'il développe un univers partagé de livre en livre. Mais la tentation occasionnelle de réunir des protagonistes d'œuvres jusque-là disjointes — on l'a vu avec Berthe Bovary, Bouvard et Pécuchet - suggère que, créés par un même écrivain, ils appartiendraient à un imaginaire commun et n'attendraient que le coup de pouce d'un continuateur un peu imaginatif pour se rencontrer. D'autres croisements transfictionnels, plus résolument ludiques, se privent manifestement d'une caution préalable et se donnent comme de purs gestes combinatoires. Bien implantée dans le domaine de la fan fiction sous le nom de crossover, cette pratique aboutit à des alliages souvent étonnants, comme ceux entre James Bond et les Pokemons (GlassDragonflyOfDeath, 2012) ou Lizzie Bennet et Doctor Who ${ }^{3}$.

La conception de la fiction qui sous-tend ces croisements est moins centrée sur l'intrigue (qui est rarement l'aspect le

\footnotetext{
${ }^{3} \mathrm{Au} 31$ mai 2015, neuf récits proposent cette formule sur le site Fanfiction.net, $<$ https://www.fanfiction.net/Doctor-Who-and-Pride-and-PrejudiceCrossovers/21/3624/>.
} 
mieux réussi de ces récits) ou sur sa dimension rhétorique que sur l'idée de combinatoire : une conception ludique qui fait de l'imaginaire un vaste réservoir où les frontières importent moins que les appariements, les structures narratives moins que les miracles ponctuels. Tout cela semble éminemment postmoderne, mais la formule était déjà pratiquée dans l'antiquité et à l'époque classique (sous le nom de contamination) et, plus près de , au XIXe siècle dans des romans comme les Voyages de Saturnin Farandoul (Robida, 1879), qui réunit quantité de personnages verniens, ou The Pursuit of the House-Boat (Bangs, 1903), où les enfers sont le lieu de résidence des personnages historiques, mythologiques et fictionnels. On la retrouve aussi chez des écrivains peu suspects de postmodernisme, comme Michel Tremblay, dont la pièce Les Héros de mon enfance (1976) regroupe les personnages de divers contes: le Petit Chaperon Rouge, la fée Carabosse, Cendrillon, le Petit Poucet, le Loup, le Prince Charmant, etc. Il s'agit chaque fois de faire de l'imaginaire, ou de telle de ses portions (l'imaginaire des contes de fées, celui de Jules Verne, etc.), un vaste territoire dont le découpage des œuvres ne parviendrait pas tout à fait à masquer l'unicité. L'apport de la postmodernité consiste surtout à donner une portée maximale à de tels jeux dans lesquels elle invite à voir, non plus d'amusantes tentatives isolées, mais un principe général de la fiction.

L'ambivalence des croisements, ici fondés sur une continuité diégétique présumée, visant là le choc des rencontres incongrues, caractérise en fait l'ensemble du domaine transfictionnel. Promettant tacitement ou explicitement des 
révélations, les textes transfictionnels jouent sur deux tableaux à la fois : la mise au jour des replis d'un univers fictif (l'avenir des personnages, leurs pensées secrètes, etc.) et l'irruption d'une nouveauté produite par l'écriture : le non-dit et l'inédit, en somme. Les lecteurs de ces récits supposent, ne serait-ce que provisoirement et par jeu, l'existence d'une diégèse dans laquelle originaux et dérivés effectueraient des ponctions complémentaires. En même temps, ils savent bien que les divulgations transfictionnelles sont le fruit d'une interférence scripturale: même les plus enclins à accepter l'illusion référentielle ne peuvent ignorer que les transfictions fabriquent de la fiction avec de la fiction.

Ce que la transfictionnalité révèle, au bout du compte, c'est la complexité du domaine dans lequel elle s'inscrit. Déploiement simultané d'un récit, d'une perspective et d'un cadre qu'on voudrait étendre aux dimensions d'un monde, la fiction est une pratique enchevêtrée, que la multiplication des œuvres autour d'une "même » diégèse ne simplifie en rien. La lecture de ces œuvres oscille entre illusion et distance, circonspection devant des manœuvres cousues de fil blanc et excitation à l'idée de découvrir des versants inconnus d'un personnage, d'une histoire ou d'un univers imaginaire. Régulièrement privées de début, constamment segmentées, pas toujours racontées jusqu'à leur terme, les existences fictives exercent une fascination que plusieurs voudraient renouveler, poursuivre ou approfondir. Les transfictions ne satisfont ce désir qu'en confrontant leurs propres lecteurs à de nouvelles questions. Elles ne «comblent » qu'en dessinant de nouvelles zones d'ombre, ne prolongent des récits qu'en s'aventurant sur des terrains jusque-là forclos, ne «révèlent» qu'en faisant réaliser à quel point l'idée même de révélation est 
problématique lorsqu'il s'agit de fiction. La lumière à la fois séduisante et équivoque qu'elles jettent n'en est que plus précieuse.

\section{Bibliographie}

AMÉRY, Jean. (1991 [1978]), Charles Bovary, médecin de campagne, trad. de l'allemand par Françoise Wuilmart, Arles, Actes Sud.

BAKER, Jo. (2013), Longbourn, Toronto, Vintage.

BANGS, John Kendrick. (1903 [1897]), The Pursuit of the HouseBoat, New York, Harper \& Brothers.

BARING-Gould, W. S. (1967), The Annotated Sherlock Holmes, vol. 2, New York, Clarkson N. Potter.

BAYARD, Pierre. (1998), Qui a tué Roger Ackroyd?, Paris, Minuit, coll. « Paradoxe».

Benoît-Jeannin, Maxime. (1991), Mademoiselle Bovary, Paris, Balland.

Billot, Antoine. (2006), Monsieur Bovary, Paris, Gallimard, coll. « L'un et l'autre ».

BORGES, Jorge Luis. (1957 [1941]), «Examen de l'œuvre d'Herbert Quain », trad. de l'espagnol par P. Verdevoye, dans Fictions, Paris, Gallimard, coll. « Folio ».

Chaulet, Georges. (1962), Fantômette contre le hibou, Paris, Hachette, coll. « Bibliothèque rose ».

Christie, Agatha. (1948 [1926]), The Murder of Roger Ackroyd, Harmondsworth, Penguin, coll « Mystery and Crime »

Coetzee, J. M. (1988 [1986]), Foe, trad. de l'anglais par Sophie Mayoux, Paris, Seuil. 
CONAN Doyle, Arthur. (2006a [1893]), " Le dernier problème ", dans Les Aventures de Sherlock Holmes, trad. de l'anglais par Éric Wittersheim, vol. 2, Paris, Omnibus.

-. (2006b [1903]), "La maison vide», dans Les Aventures de Sherlock Holmes, trad. de l'anglais par Éric Wittersheim, vol. 2, Paris, Omnibus.

DaOud, Kemel. (2014), Meursaut, contre-enquête, Arles, Actes Sud.

DibDIN, Michael. (1978), The Last Sherlock Story, New York, Pantheon.

DuboIS, Jacques. (2011), Figures du désir. Pour une critique amoureuse, Bruxelles, Les Impressions Nouvelles, coll. «Réflexions faites».

EchenOZ, Jean. (1997), Un an, Paris, Minuit.

- (1999), Je m'en vais, Paris, Minuit.

ESTLEMAN, Loren D. (2000 [1978]), Sherlock Holmes vs. Dracula, or: The Adventure of the Sanguinary Count, New York, ibooks.

Flaubert, Gustave. (1986 [1957]), Madame Bovary, Paris, Garnier-Flammarion, coll. « GF ».

FouCAult, Michel. (1988 [1963]), Naissance de la clinique, Paris, PUF, coll. « Quadrige ».

Genette, Gérard. (1982), Palimpsestes, la littérature au second degré, Paris, Seuil, coll. « Points ».

GLASSDRAgonflyOFDEATH. (2012), «The Name's Giratina, Codename $007 », \quad$ FanFiction.net, <https://www.fanfiction.net/s/8713375/1/The-Name-sGiratina-Codename-007>.

Grimaldi, Laura. (1995 [1991]), Monsieur Bovary, trad. de l'italien par Geneviève Lambrich, Paris, Métailié. 
MARTIN, Valerie. (1991 [1990]), Mary Reilly, New York, Pocket.

MooRe, Alan et Kevin O'Neill. (2002), The League of Extraordinary Gentlemen, Boston, DC Comics.

PARSONS, Terence. (1980), Nonexistent Objects, Hew Haven, Yale University Press.

PInGET, Robert. (1962), L'Inquisitoire, Paris, Minuit.

—. (1987), L'Ennemi, Paris, Minuit.

RHYS, Jean. (1982 [1966]), Wide Sargasso Sea, New York, Norton.

RoBIDA, Albert. (1879), Voyages très extraordinaires de Saturnin Farandoul dans les cinq ou six parties du monde et dans tous les pays connus et même inconnus de monsieur Jules Verne, Paris, Librairie illustrée.

RoSE, Jacqueline. (2002 [2001]), Albertine, Londres, Vintage.

SAINT-GELAIS, Richard. (2012), Fictions transfuges: la transfictionnalité et ses enjeux, Paris, Seuil, coll. « Poétique ».

THÉRIEN, Gilles. (1984), "Sémiologie du discours littéraire», Recherches sémiotiques, vol. 4, no 2, p. 155-176.

Tremblay, Michel. (1976), Les Héros de mon enfance, Montréal, Leméac, coll. « Théâtre ».

—. (1992), Marcel poursuivi par les chiens, Montréal, Leméac, coll. « Théâtre ». 